\title{
Assuring quality of color graphics for aerospace interfaces
}

\author{
Larry Arend \\ NASA Ames Research Center, Moffett Field, CA, 94035
}

\begin{abstract}
Aerospace information displays require some form of quality assurance due to public safety concerns. However, the color design methodologies, standards, and guidelines employed in development of earlier displays are insufficient to guide current designs, which have reached map-like complexity. For these displays designers can only assure legibility and good attention management with designs that consider relations among all of the graphic elements. Quality assurance needs to focus on performance in context rather than graphic elements. We are investigating web-based color-guidance tools to help advance the design and review processes. Individual performance issues that guidance statements address are demonstrated in the context of a hyperlinked network of supporting information at varying levels of technical detail. Draft color designs can be developed and modified in this environment using web-based design tools that visualize the consequences of particular choices and the interactions among the chosen colors.
\end{abstract}

Keywords: color standards, color guidelines, aerospace, color graphics

\subsection{THE PROBLEM}

The quality and usability of aviation information displays need close scrutiny due to the public safety implications. Designs undergo extensive development and testing before receiving Federal Aviation Administration certification for operational use.

Improvements in communications, computing, and displays now allow visualizations with map-like complexity (e.g. Fig. 1). These displays can improve the situation awareness and reduce the cognitive workload of aerospace operators, but the complexity has a price. The increased information density poses unfamiliar and difficult challenges for the designers of the graphics and for those charged with certifying their safe use ${ }^{1}$. For these displays it is not sufficient to set standards for individual graphic elements. Designers can assure legibility and good attention management only with a coordinated, unified design that considers relations among all of the graphic elements. To achieve this designers need deeper technical understanding of applied vision than previously.

One of the biggest challenges is color assignments. In older displays small quantities of stroke graphics (lines, text, symbols) were displayed against uniform dark backgrounds or backgrounds with a few fixed, stationary colors. In newer, "moving map" style displays the stroke graphics move relative to area graphics (i.e., terrain and weather), and the area graphics move relative to each other.

The complexity of these new designs requires a new approach to color design methodologies, standards, and guidelines. Those employed in development of earlier displays either don't apply to or are insufficient to guide current designs.

\subsection{CANDIDATE SOLUTIONS}

What are the main options for assuring usable color designs in safety critical applications?

One could hire experts in applied color science and give them controlling roles in the processes of development and regulatory review. There are two problems with this approach. First, finding the right color experts would be a challenge. While there are many color experts worldwide, only a handful have the necessary combination of specialized training, experience, and interest in actual management of information display development. But even if one could find 
the right people, experience in aviation technology and other fields indicates that this choice is unlikely to be made due to engineering cultural factors ${ }^{2}$. In most developments of display hardware and software it has been difficult to bring appropriate color experts and system developers together. This has been partly due to underinvestment (time and money) in the design phase of software projects (if there is a distinct design phase at all) and partly due to the management customs of the engineering community.

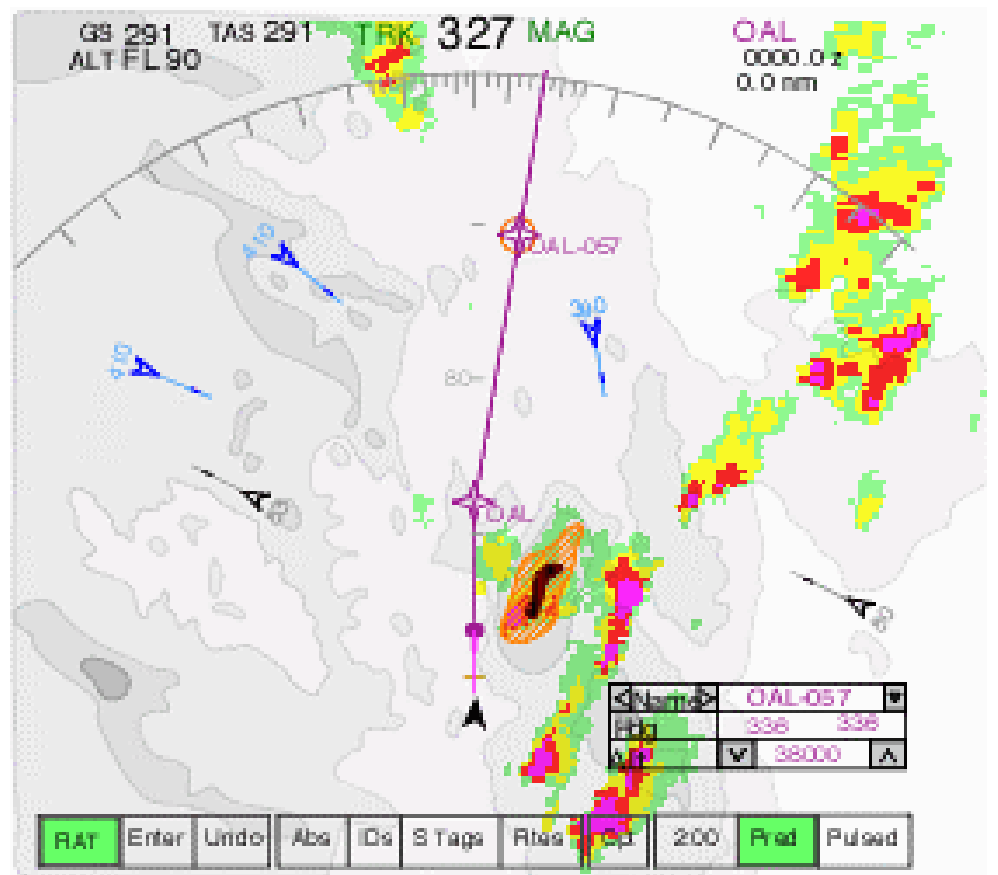

Fig. 1. A prototype cockpit display of hazards. [Note: The figures in this paper are reproduced in color in the electronic copy of the document, available on the proceedings CD or from the author: larend@ mail.arc.nasa.gov.]

Another approach would be to leave hardware and software engineers in control and require and assist them to obtain adequate applied color training. Currently short courses and printed materials are the main resources available for this approach.

Applied color experts have offered a number of short courses, both in conjunction with professional meetings and as intensive summer courses at universities. The best of these courses provide rigorous introduction to the concepts and methods of color engineering and perception. Nevertheless, it is unreasonable to expect that they can be a substitute for extended graduate level education in vision science and experience with applying that education to a variety of problems in color design projects. The usability problems encountered in design of rich graphic displays involve complicated tradeoffs among graphic choices. Recognizing the causes and potential solutions of problems can involve subtleties that challenge even color experts with extensive education and experience.

A number of books have been directed at color design for electronic display ${ }^{3}$. In spite of some excellent content, printed materials generally lack sufficient color graphic capability to demonstrate color principles. The technical demands and cost of high-quality color reproduction are high and the markets are relatively small. Due to their fixed format printed materials also frequently fail to bridge the gaps between the designer's specific problems and general principles of display technology and applied color perception. 
The last approach is similar to the current system. Design would continue to be controlled by hardware and software engineers with limited color expertise. Quality assurance would be provided by a system of standards and guidelines that new information displays would have to meet to be certified as safe.

With older, simple displays this approach produced acceptable results, but for newer, more complicated displays conventional standards are insufficient to deal with the complex design issues. Limitations of current color standards are of several kinds.

Some are too vague to be useful to a designer with limited applied color training and experience. For example, "The contrast ratio (total foreground luminance/total background luminance) shall be sufficient to provide a promptly discernible, easy to read image under all conditions of cockpit illumination and under all conditions of eye adaptation to the external scene."

"The display luminance shall be sufficient for the display to perform its intended function."

"Symbol quality characteristics shall not cause erroneous interpretations..."

"Lines, symbols, and characters shall be clear, legible, and unambiguous, at all times within the viewing envelope."

The goals are obviously desirable, but can be difficult to achieve in a complicated design.

Others are more clear, but too simple and rigid to deal with varying graphic contexts. For example,

"The average white contrast ratio over the usable display surface should be a minimum of 20:1 at the design eye position..."

"Colors shall be selected from the following set of nine: Red, Tan/Brown, Amber, Yellow, Green, Cyan, Blue, Magenta, and White.",

"...the average white symbol luminance ...should be at least $257 \mathrm{~cd} / \mathrm{m} 2 . . . " 10$

"The contrast requirements for symbol legibility and color identification are somewhat in conflict. Increased luminance contrast results in increased symbol legibility but decreased color discriminability. However a balance may be achieved that achieves both good legibility and enhances color discrimination. For example, a symbol to background luminance contrast ratio of about 20:1 would generally yield very good symbol legibility as well as good color discrimination and identification."11

While it might seem at first glance that requiring high luminance contrast would be universally desirable, in complex displays it is often better for some of the information to be barely legible (Fig. 2).

Guidelines and standards statements tend to be of two kinds, those relating to low level graphics features and those relating to the utility of the overall graphic. The usability of advanced graphics depends on the interrelationships among the graphic elements, with design choices depending on multiple contexts. To deal with these circumstances standards and guidelines should probably be framed in terms of performance specifications rather than graphic specifications. For example, "Indications of traffic hazards shall be more salient than non-hazardous information" would be more

universally relevant than "Symbols for hazardous traffic shall have luminance contrast of 20:1 or greater." Some existing standards concentrate on performance:

"Displayed information shall have sufficient luminance contrast and/or color difference to discriminate between the following as applicable:

a) Between symbols, characters and/or lines and overlaid background (ambient or generated).

b) Between individual symbols, characters and lines when they overlay ambient or generated backgrounds.

c) Between generated backgrounds and ambient backgrounds.

d) Between generated backgrounds of various specified colors.

In all cases the luminance contrast and/or color differences between all symbols, characters, lines, or all backgrounds shall be sufficient to preclude confusion or ambiguity as to information content of any displayed information." 12

These are fine goals, but how are they to be achieved? The problem with performance standards is that design engineers need help to know how to achieve them and certifiers need help to recognize whether they are adequately met.

\subsection{RESEARCH APPROACH}

We are investigating a new kind of tool for guidance of color design that may help address these challenges. This webbased suite of information and tools ${ }^{13}$ is intended to support movement toward manageable performance-based standards. 

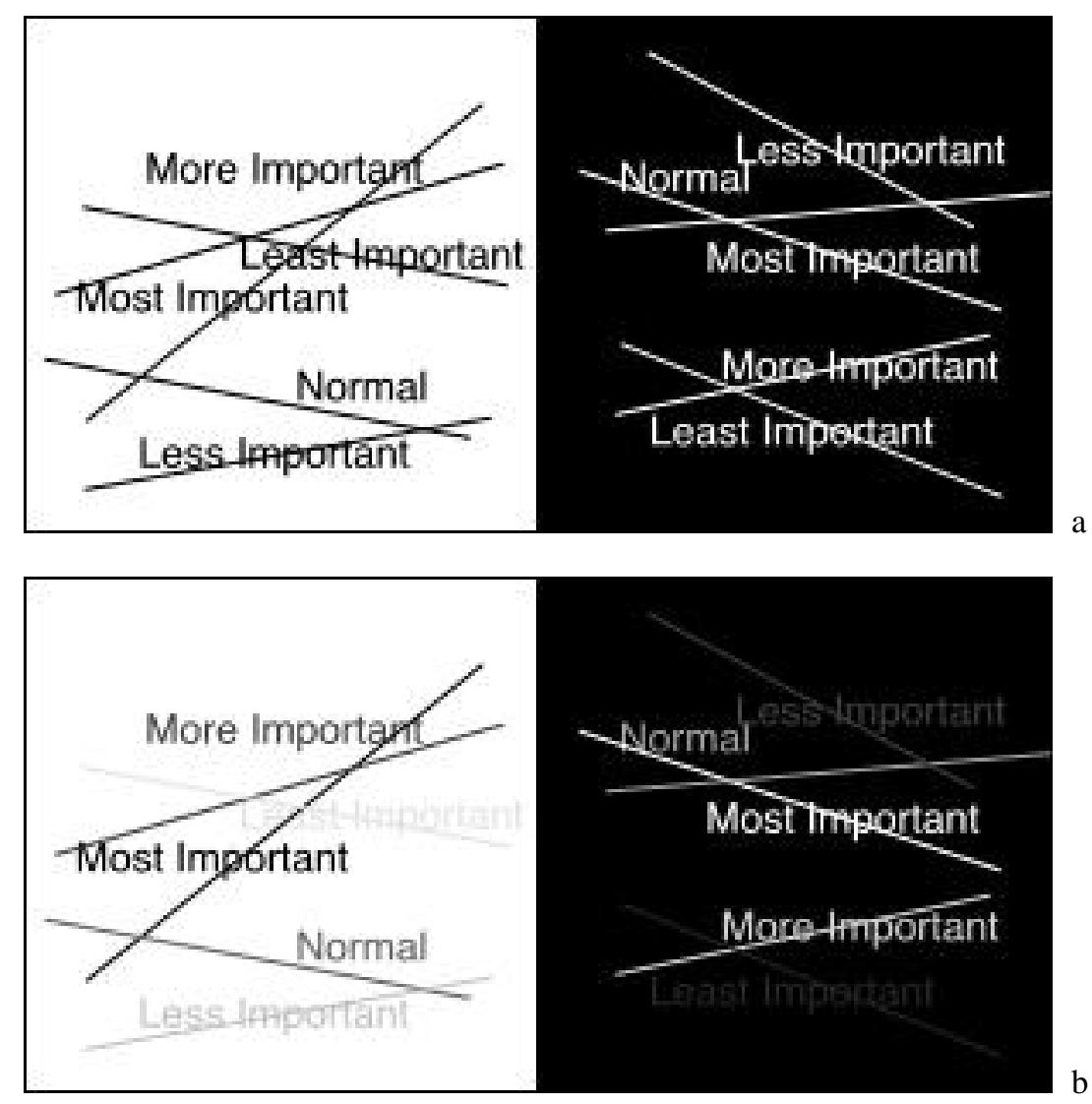

Figure 2. a) Giving all elements the same, high contrast allows the less important information to interfere with attention to the more important. b) Giving less important, context information lower contrasts allows the high importance information to stand out without losing the context. [Note: The figures in this paper are reproduced in color in the electronic copy of the document, available on the proceedings CD or from the author: larend@ mail.arc.nasa.gov.]

We designed the website for the use of non-color-experts with technical backgrounds, the engineers that develop and review aerospace information graphics. It includes tutorial material, reference material, and some interactive design tools. In its current form it includes:

1) An annotated checklist for design of a color graphics page, with links to tutorial material on each step and component of the design process.

2) Step-by-step examples of design of two displays, an integrated hazard display for the cockpit and the corresponding display for air traffic controllers.

3) Tutorial material on color guidelines, standards, and requirements with discussion and examples of the value and limitations of the current styles of guidelines. It lists current standards and guidelines that include color usage elements.

4) Supporting tutorial material on applied color vision concepts, with pointers to the underlying color science.

5) Interactive color selection tools that show the display's available colors within a perceptual color space and allow immediate preview of the selected color combinations.

The web medium has two immediate advantages: a) we can use its hypermedia capabilities to organize the content in several different ways simultaneously and interrelate the several kinds and levels of detail of information about color usage and b) we can exploit the capability of the users' desktop computers to display numerous high-quality color illustrations. 


\section{Annotated checklist}

The design of the site provides for several alternative ways to enter and use the site. The annotated checklist provides relatively new users with a structured view of the role of color design in the overall display design process. It takes the user through the major choice points in the design, starting with organization of the data types into a hierarchy of urgency (Table 1) and then leading the user through the graphics decisions necessary to give the data elements the saliency appropriate to their place in that hierarchy (Fig. 3). For each decision there are links to the relevant tutorial material on other pages of the site. Those supporting materials are organized to make clear the various options the designer might choose at that point, the implications for usability, and the implications for the other design choices that must be made in completing the design.

\section{Sample ATC Data Hierarchy}

1. Urgent aircraft (approaching hazard, declared emergency)

2. Hazard area variables (weather, terrain)

3. Focal aircraft ("own" aircraft)

4. Context aircraft (not hazard for "own" aircraft)

5. Context lines, symbols (boundaries, airways, navaids)

6. Context area variables (own vs. other sectors) red aircraft symbols red, yellow blobs high luminance-contrast aircraft symbols low luminance-contrast aircraft symbols faint gray lines, text gray background shading

Table 1. A sample urgency hierarchy for an air traffic control display.

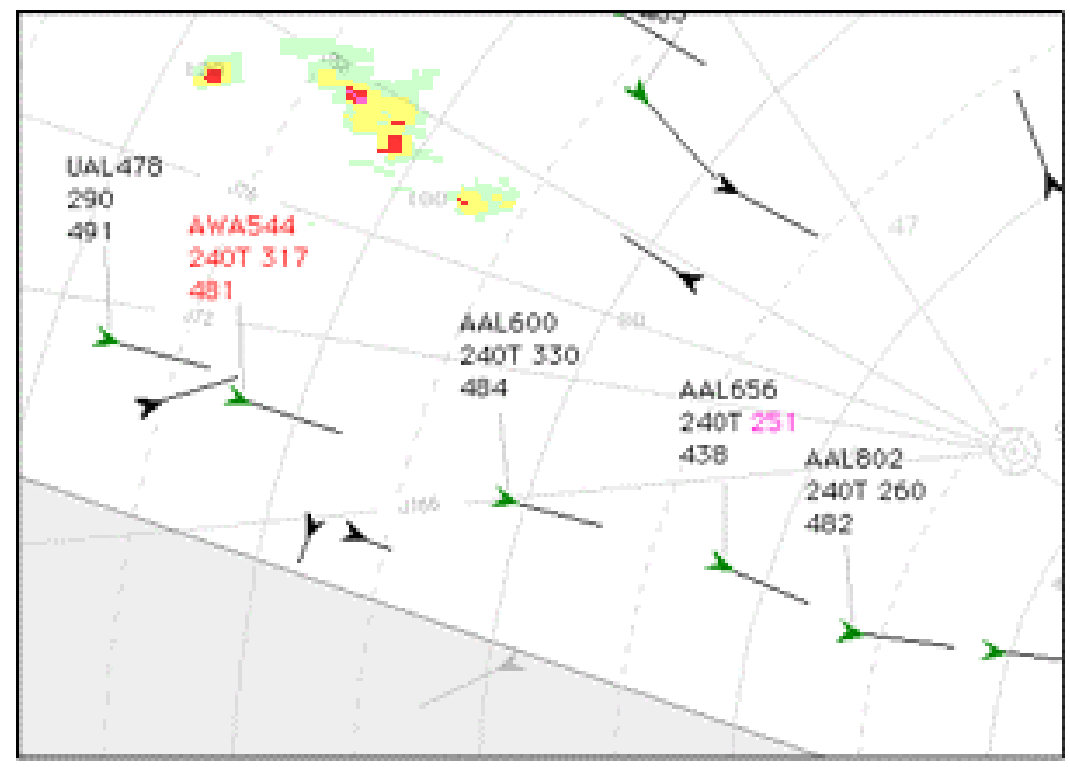

Figure 3. Air traffic control display design that implements a saliency hierarchy corresponding to the urgency hierarchy of Table 1. [Note: The figures in this paper are reproduced in color in the electronic copy of the document, available on the proceedings CD or from the author: larend@ mail.arc.nasa.gov.]

\section{Aerospace design examples}

This section of the site gives two step-by-step examples of the process laid out in the annotated checklist. One shows the decision stages in design of an integrated hazard display for the cockpit. The other illustrates design of the 
corresponding display for air traffic controllers. These examples make the steps of the design process less abstract by using each stage of the checklist and the linked supporting material.

\section{Tutorial on color guidelines, standards, and requirements}

This section presents a discussion of current aerospace color guidelines, standards, and requirements with examples of the value and limitations of the current styles of guidelines. Several groups of dedicated applied-color experts have put an enormous amount of effort over many years into development of these guidelines. While the quality and form of the guidelines vary widely, most cover a relatively small set of topics. Each of these recurring topics is discussed generically in the site, with links to tutorial material on the related applied color concepts. Most of the guidelines fall into the categories of concerns about sufficient luminance-contrast for symbols and concerns about discrimination and identification of colors used to label symbol classes. Only a few address global design issues like control of the user's attention or interference (masking) among symbols or textured backgrounds. Sample guidelines are illustrated by examples of cases where the guideline applies and cases where it does not. For example, Fig. 4 shows both cases with respect to the frequent warning against use of pure blue. The site includes a list of current standards and guidelines that include color usage elements.

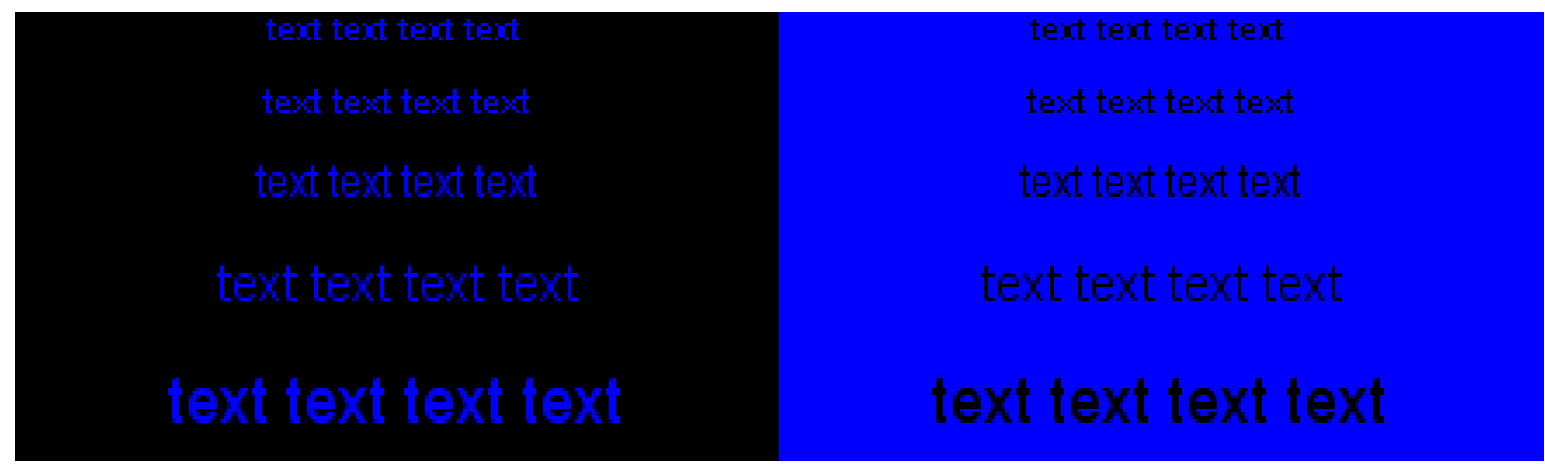

text text text text

text text text text

text text text text

text text text text

text text text text text text text text

text text text text

text text text text

text text text text

\section{text text text text}

Fig. 4. Pure blue symbols are problematic against a black background, but not against white. The same applies to the reverse, blue backgrounds for black and white text. [Note: The figures in this paper are reproduced in color in the electronic copy of the document, available on the proceedings CD or from the author: larend@ mail.arc.nasa.gov.]

\section{Tutorial on applied color vision concepts}

Applied color vision concepts, with links to underlying color science, are discussed as background material for the graphics topics. To help users see better the relevance of the materials to color graphics design, the material is grouped in relation to behavioral topics rather than by theories of physiological mechanisms, the more usual organization in modern vision science. At this stage of development of the site the treatment is intended less as a thorough coverage of vision science than as a demonstration to the users that there is a structured science of human vision that can inform 
graphic design. Pointers and links are provided to guide interested users to more detailed information from other sources.

\section{Color selection tools}

Once designers understand the desired relations among colors in their design, they still need to choose particular colors. Color experts may be able to readily relate the desired visual properties of colors to the color spaces of computer graphics, but less experienced designers need tools that can help them visualize the consequences of particular choices and the interactions among the chosen colors. The site includes two simple tools for this purpose. The current version of the interactive color selection tools show the user the display's available colors within a perceptual color space. The tools allow immediate preview of the color combinations as they are selected.

The tools, like the demonstrations elsewhere in the site, exploit the near universal availability of high quality color displays on the designers' desktops. At present a generic desktop display is assumed, but methods are under development to let the users fine tune the presentation of the site to their individual desktop displays. Each of the two tools shows the gamut of the monitor in a hue/chroma/lightness space in one of two organizations. The space chosen ${ }^{14}$ is CIE $L^{*} C^{*}{ }_{u v} h_{u v}$. In one tool the user selects the desired lightness level (L*) for the next color pick, resulting in display of the range of hues $\left(h_{u v}\right)$ and chromas $\left(C^{*}{ }_{u v}\right)$ available on the monitor at that lightness. In the other organization the user first picks a hue on a hue circle, resulting in display of a two-dimensional array of patches showing all chromas available at ten lightnesses ranging from the monitor's black to the monitor's white (Fig. 5). In either case the chosen color is then shown in a separate display as a symbol or background color against all previously chosen colors (Fig. 6).

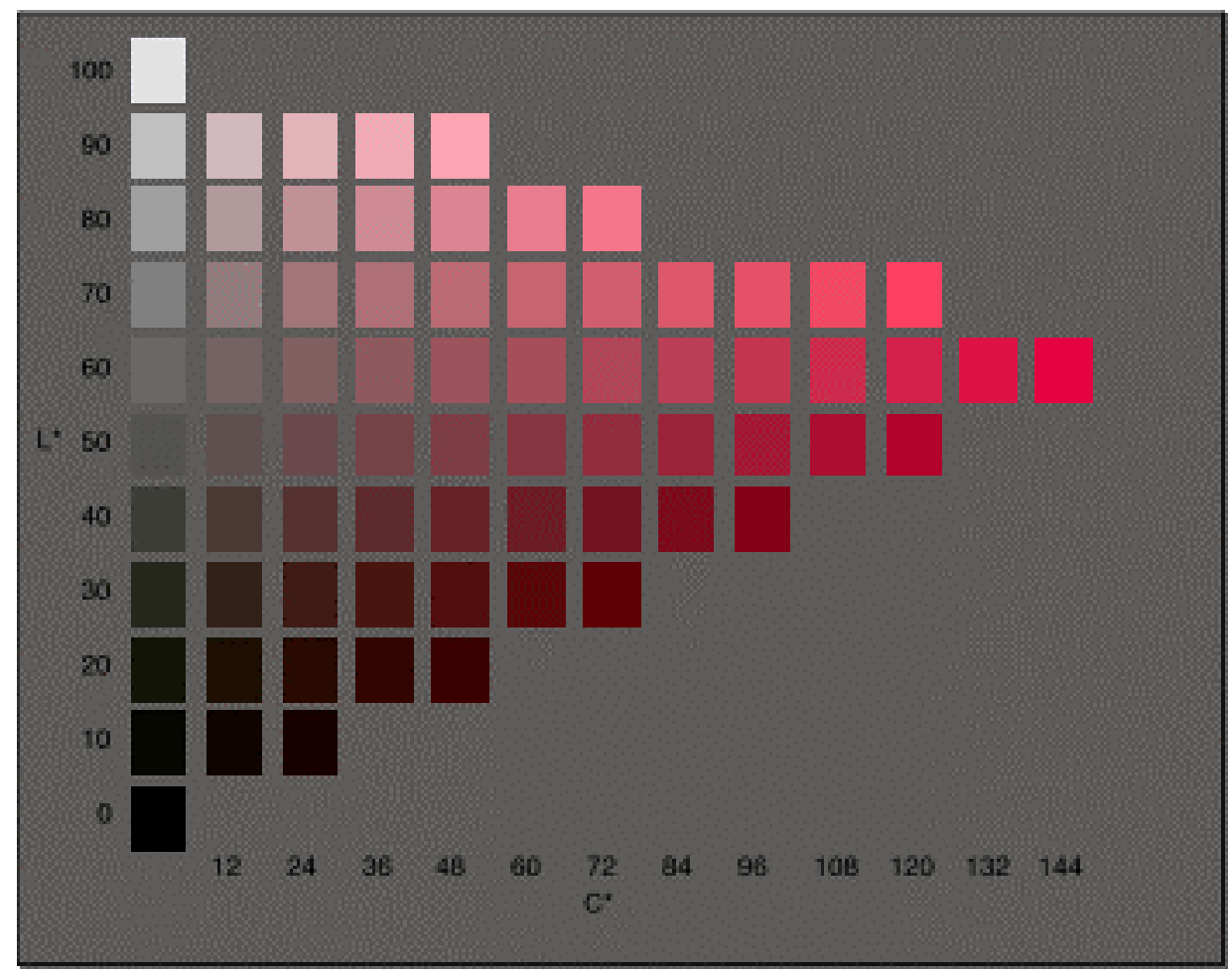

Figure 5. The color gamut available at a red hue. [Note: The figures in this paper are reproduced in color in the electronic copy of the document, available on the proceedings CD or from the author: larend@ mail.arc.nasa.gov.]

\section{General considerations}

In developing the content of the site we applied several constraints. Given our assumptions about our targeted users the top level pages were designed to be intelligible to engineers familiar with display technology. Content at this top level 
uses terms and concepts from the physics of light and basic vision, but does not assume familiarity with perceptual and cognitive color phenomena. More technical applied perceptual information appears in supporting pages, but basic research is mostly addressed through pointers to external sites.

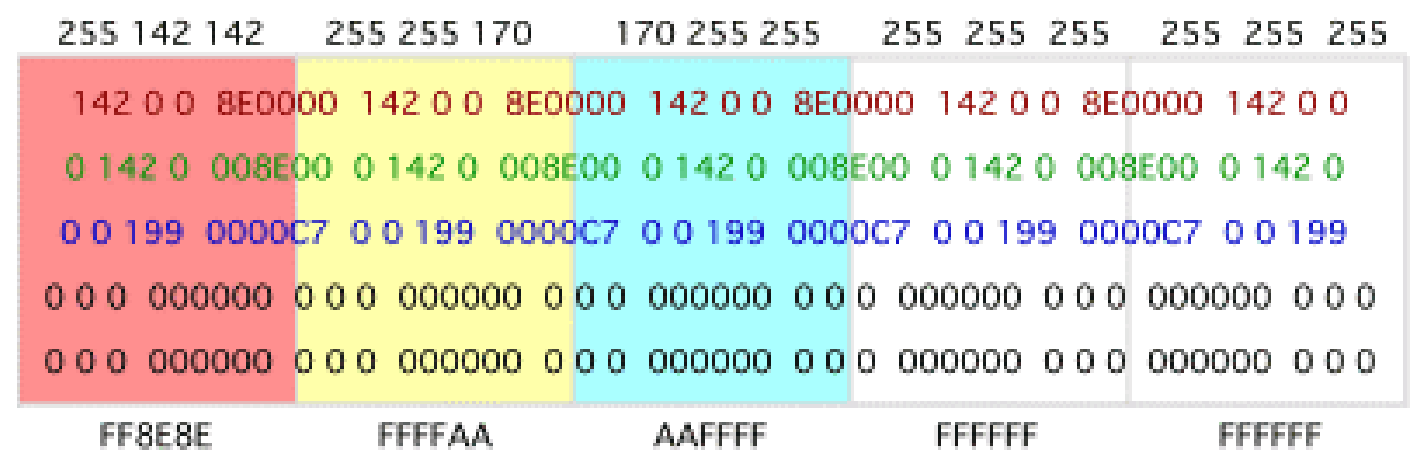

Figure 6. The set of selected symbol colors is displayed against the set of selected background colors. [Note: The figures in this paper are reproduced in color in the electronic copy of the document, available on the proceedings CD or from the author: larend@mail.arc.nasa.gov.]

We also restricted perceptual topics to those that could be directly demonstrated with graphics. The logic behind this restriction is that phenomena which are not strong enough to be reliably demonstrated on the user's desktop display are probably not first order issues in the applications the user is designing. This ignores or minimizes treatment of some issues that have traditionally been included in applied color research, but we felt that this restriction is outweighed by the contribution to user confidence provided by the direct demonstrations.

\section{Tests of designs}

The goal of the site is to help non-color-expert designers of interfaces generate better initial designs and to help them think systematically about the usability consequences of their design choices. Its successors may also enable color standards based on performance to guide both designers and safety certifiers. It is not intended as a substitute for consultation by color experts or safety testing. As in the current system, the usability and safety of designs requires evaluation in high-fidelity simulations and field tests. The high cost (time and money) of this testing can be reduced by starting with well-developed designs.

\section{ACKNOWLEDGEMENTS}

This research was sponsored by the NASA Aerospace Operations Systems Program and the NASA Advanced Air Transportation Technologies Project.

\section{REFERENCES}

1. Society of Automotive Engineers, ARP5108 Human Interface Criteria for Terrain Separation Assurance Display Technology, SAE, Warrendale, Pennsylvania, 1997, pp. 14-15.

2. C. E. Billings, Aviation Automation: The Search for a Human-Centered Approach, Lawrence Erlbaum Assoc., Mahwah, NJ, 1997.

A. Cooper, The Inmates are Running the Asylum, Macmillan Computer Publishing, Indianapolis, 1999.

D. A. Norman, Things that Make Us Smart: Defending Human Attributes in the Age of the Machine, AddisonWesley, Reading, MA, 1993.

3. For example, H. J. Durrett, Color and the Computer, Academic Press, San Diego, 1987. 
L. G. Thorell, \& W. J. Smith, Using Computer Color Effectively: An Illustrated Reference. Englewood Cliffs, New Jersey, Prentice Hall, 1990.

4. Society of Automotive Engineers, ARP4256 Design Objectives for Liquid Crystal Displays for Part 25 (Transport) Aircraft, SAE, Warrendale, Pennsylvania, 1996, p. 16.

5. Society of Automotive Engineers, AS8034 Minimum Performance Standards for Airborne Multipurpose Electronic Displays, SAE, Warrendale, Pennsylvania: 1993, p. 11.

6. Society of Automotive Engineers, ARP4256 Design Objectives for Liquid Crystal Displays for Part 25 (Transport) Aircraft, SAE, Warrendale, Pennsylvania, 1996, p. 8.

7. Air Std 10/72, Electronic Colour Display Systems, 1990, p.4.

8. Society of Automotive Engineers, ARP4256 Design Objectives for Liquid Crystal Displays for Part 25 (Transport) Aircraft, SAE, Warrendale, Pennsylvania, 1996, p. 16.

9. Society of Automotive Engineers, ARP4102 Core Document, Flight Deck Panels, Controls and Displays, SAE, Warrendale, Pennsylvania, 1994, p. 11.

10. Society of Automotive Engineers, ARP4256 Design Objectives for Liquid Crystal Displays for Part 25 (Transport) Aircraft, SAE, Warrendale, Pennsylvania, 1996, p. 12.

11. Society of Automotive Engineers, ARP4102 Core Document, Flight Deck Panels, Controls and Displays, SAE, Warrendale, Pennsylvania, 1994, p. 7.

12. Society of Automotive Engineers, AS8034 Minimum Performance Standards for Airborne Multipurpose Electronic Displays, SAE, Warrendale, Pennsylvania: 1993, p. 7.

13. L.Arend, NASA Color Usage Site: CD Version, Moffett Field, CA, 2002. Available from the author: larend@mail.arc.nasa.gov.

14. G. Wyszecki \& W. S. Stiles, Color Science - Concepts and Methods, Quantitative Data and Formulae, John Wiley \& Sons, New York, 1982. 